\title{
Semi-Dense And Concentrated Fullerene-Based Composites: Microdeformation and Strain Gradient Effects
}

\author{
G. Augusti \\ Dipartimento di Ingegneria Strutturale e Geotecnica \\ Università di Roma “La Sapienza”, I-00184 Roma, Italy \\ P. M. Mariano \\ DICeA, Università di Firenze, I-50139 Firenze, Italy \\ paolo.mariano@unifi.it \\ F. L. Stazi \\ SKY Italia, I-20138 Milano, Italy
}

\section{SUMMARY}

We discuss primary issues towards the multifield continuum modeling of elastic fullerene-based composites.

\section{INTRODUCTION}

Fullerenes are carbon micro-aggregates with closed-cage structure made of pentagons or hexagons. They are obtained through condensation of carbon vapor at different values of temperature and pressure, values that determine the geometry of the resulting structure. Their importance in technology is currently increasing due to a wide class of possible applications allowed by their mechanical and chemical properties.

Typically, fullerene-based composites are sol-gel structures. When they are characterized by polymeric matrices, they may combine the optical and electronic properties of semiconductors with the mechanical features of polymers [1].

Fullerene-based composites may have inorganic or/and organic nature. Among them we may select for example

- Fullerene/SiO

- Fullerene/SiO $-\mathrm{TiO}_{2}$, 
- MEH-PPV, i.e., poly[2 metoxy, 5-(2'-ethylexyloxy)-1,4-phenylene-vinylene],

- PCBM, i.e., $[6,6]$-phenylene $\mathrm{C}_{01}$-bytyric acid methyl ester,

- Phosphate - Glass - $\mathrm{C}_{60}$,

- MDMO-PPV, i.e., poly (2-methoxy-5-(3',7'-dimethylo ctyloxy)-1,4-phenylene vynilene).

Commonly, they are used for thin films, but they may be also utilized in different devices of nanotechnology as 'small" bars etc.

Prominent special properties are photoinduced electron transfer and photoluminescence coupled with the mechanical properties of polymeric elongated molecules $[1,2,3]$.

When we consider a composite made of fullerenes scattered throughout a matrix, we have a complex material in which every material element can be considered a system, a "box" containing one or more fullerene molecules, in general a family. Moreover, when the matrix has polymeric structure, the picture of the material element becomes more articulated because families of polymeric molecules are mixed with the fullerene structures. An appropriate continuum model of such a material cannot consider only the deformation due to the change of relative placement between neighboring material elements; we should consider in addition what happens within each material element. To this aim we introduce a coarse grained morphological descriptor (order parameter) of the substructure induced by the macromolecular nature of the material element. In this way we put ourselves in the general setting of multifield theories describing the influence of material substructure on the gross mechanical behavior of bodies $[4,5,6]$. By following the general framework of multifield theories we need basically to select an order parameter, an explicit form of the free energy, and a dissipation pseudo-potential when we deal with irreversible processes (a circumstance not treated here). Various choices can be made in the case treated here.

1. Since fullerene molecules are characterized by hexagonal or pentagonal symmetries, they may induce quasi-periodic structures within the body, then the propagation of elastic waves may be correctly described taking into account not only phonon degrees of freedom (the standard ones of elastic waves in perfectly crystalline materials), but also of phason degrees of freedom like in quasicrystalline materials. The latter ones are described by a "microdisplacement", i.e., a vector attached at each point $\mathrm{X}$ describing the local rearrangement between fullerene molecules and matrix to restore periodicity.

2. We may also consider as a prominent feature the circumstance that the fullerene macromolecules have a stiffness different from the one of the surrounding matrix, so that we may select as order parameter a second order symmetric tensor. It describes at each point $\mathrm{X}$ the possible independent deformation of every family of fullerene molecules, within every material element, with respect to the matrix.

3. If we put attention on the property of polarizability, as it occurs in presence of polymeric matrices, a natural descriptor of the substructural state is the vector of polarization.

4. If one considers strictly only the polyedral structure of fullerene molecules, by following a conjecture of Sir Michael Atiyah (see, e.g., [7]), we may select as morphological descriptor an element of $\mathrm{U}(n) / \mathrm{U}(1)^{n}$, 
where $n$ is the number of atoms of the typical molecule, and $U(n)$ is the group of unitary $n \times n$ matrices.

The elements of $\mathrm{U}(n) / \mathrm{U}(1)^{n}$ are $n$ orthonormal vectors in $\mathrm{C}^{n}$, defined up to a phase (see [7]).

5. We may finally use a spinor representation of each macromolecule.

Other choices can be adopted in principle. We work below on item 2 because in this way (i) we may suggest a manner to obtain explicit constitutive relations from lattice models, and (ii) we may put in evidence the possible occurrence of strain gradient effects in these exotic composites. We then associate to every material element its place in space and a second order tensor A describing the independent deformation of the fullerene molecules. In writing the external power of interactions, acting on an arbitrary part of the body, we account for the presence of fullerenes by taking into account interactions that measure the extra power developed in the time rate of $\mathrm{A}$ and satisfy appropriate balance equations. When the fullerene molecules are semi-dense, they can deform independently with each other; in contrast, when they are dense, they may generate gradient effects of macroscopic strain because of the presence of a natural internal constraint that makes their presence "latent".

We obtain constitutive equations from a lattice model of the material element, using an identification based on an equivalence between the discrete and the continuum in terms of power. The resulting explicit expressions of the constitutive equations depend on some internal lengths describing the dimensions of the fullerenes and the distance between neighboring molecules

\section{A POSSIBLE CONTINUUM MODEL}

Let $\mathrm{B}$ be the regular region of the three-dimensional Euclidean space $\mathrm{E}^{3}$ occupied by the body in its reference place. The material element at $\mathrm{X}$ in $\mathrm{B}$ is a "box" containing a family of fullerene molecules. Each molecule is characterized by a symmetric second-order tensor $A^{*}$ (i.e. an element of Sym) representing its deformation with respect to the surrounding matrix. A distribution function $f: \operatorname{Sym} \rightarrow \mathrm{R}$ assigns to each $\mathrm{A}^{*}$ the number of molecules described by $A^{*}$ in the material element and should depend on $A^{*}$ through its invariants (objectivity). The average $A$ of $A^{*}$ over the family characterizing each element is the order parameter selected here. Two sufficiently smooth mappings are then defined over $\mathrm{B}$ : the placement $\mathrm{x}: \mathrm{B} \rightarrow$ $\mathrm{E}^{3}$ and the order parameter $\mathrm{A}: \mathrm{B} \rightarrow$ Sym. The placement is an injective mapping and is also orientation preserving, its gradient $\nabla x$ is indicated by $F$; the current place $B^{\prime}=x(B)$ of the body is a regular region too. Motions are then defined by time-parametrized families $x_{t}$ and $A_{t}$ and we write $x(X, t)$ and $A(X, t)$ to indicate the current place and the current value of the order parameter at $t$. Rates in their Lagrangian description are indicated by $v(X, t)$ and $V(X, t)$. Among possible different rates of $A$, an important role is played by the one, indicated with $V_{R}$, which can be obtained by evaluating the action of the special orthogonal group $S O(3)$ over Sym. We then have $V_{R}=A w$, where $w(t)$ is an arbitrary rotational rate and the third order tensor $A$ is given 
by $A_{1 j k}=e_{11} A_{1 k}-A_{11} e_{1 j k}$ with e being the Ricci's permutation indicator.

We call part of $B$ any regular subregion $b$ of it with non-vanishing volume measure and use the notation < $\mathrm{g}\rangle_{b}$ and $\langle\mathrm{g}\rangle_{\partial b}$ to indicate the integrals over $b$ and its boundary $\partial b$, respectively, of the mapping $\mathrm{g}$, defined over $B$ and taking values in a linear space.

Interactions arise between material elements and are of standard and substructural nature. They are represented by the vector $b$ of bulk forces, the Piola-Kirchhoff stress tensor $T$ and the microstress tensor $S$ measuring contact interactions between neighboring material elements associated with the rate of $A$. They satisfy the standard balance of forces and the (non-standard) balance of couples given respectively by

$$
\begin{aligned}
& <\mathrm{b}>_{b}+<\operatorname{Tn}>_{\partial b}=0, \\
& <\left(\mathrm{x}-\mathrm{x}_{0}\right) \times \mathrm{b}>_{b}+<\left(\mathrm{x}-\mathrm{x}_{0}\right) \times \operatorname{Tn}+\mathbf{A}^{\mathrm{T}} \mathrm{Sn}>_{\partial b}=0,
\end{aligned}
$$

(see [6]) where $A^{T}$ means that $A_{i j k}$ becomes $\left(A^{T}\right)_{k j}$. The arbitrariness of $b$ implies from (1) the standard Cauchy's balance

$$
\mathrm{b}+\operatorname{DivT}=\mathbf{0}
$$

while, from (2), thanks also to (3), one obtains

$$
\operatorname{eTF}^{\mathrm{T}}+\operatorname{Div}\left(\mathbf{A}^{\mathrm{T}} \mathrm{S}\right)=0
$$

Note that the product $A^{\mathrm{T}} S$ is given in components by $\left(A^{\mathrm{T}} S\right)_{\mathrm{ij}}=\mathbf{A}^{\mathrm{T}}{ }_{\mathrm{ikl}} \mathrm{S}_{\mathrm{lkj}}$. The fact that $A$ is not one-to-one implies the existence of a second order tensor $Z$ such that

$$
A^{T} Z=e T F^{T}-\left(\nabla A^{T}\right) S,
$$

then, one gets from (4)

$$
A^{T}(\operatorname{DivS}-Z)=0 \text {, i.e., } \operatorname{DivS}-Z=0 \text {. }
$$

A priori constitutive restrictions can be derived from a mechanical version of the second law of thermodynamics, stating that the difference between the rate of the free energy (whose density is indicated by $\psi$ ) and the external power is lesser or equal to zero for any choice of the rates involved and for any part $b$, i.e.,

$$
\langle\psi\rangle_{b}-\left\langle\mathrm{T} \cdot \mathrm{F}^{*}+\mathrm{Z} \cdot \mathrm{V}+\mathrm{S} \cdot \nabla \mathrm{V}\right\rangle_{b} \leq 0
$$


where the dot means derivative with respect to time.

We assume first that $\psi$ has the structure

$$
\psi=\psi(\mathrm{F}, \mathrm{A}, \nabla \mathrm{A})
$$

and, then, that analogous constitutive structures are admitted by the measures of interaction, namely

$$
T=T(F, A, \nabla A), \quad Z=Z(F, A, \nabla A), \quad S=S(F, A, \nabla A)
$$

By developing the time rate of (8), the validity of the dissipation inequality (7) for any choice of the rates involved in it implies that

$$
\mathrm{T}=\partial_{\mathrm{F}} \Psi ; \mathrm{Z}=\partial_{\mathrm{A}} \Psi ; \mathrm{S}=\partial_{\nabla \mathrm{A}} \psi
$$

Once the expression of the free energy is chosen, the constitutive structure of the measures of interactions follows. In the following we restrict our attention to the elastic behavior, then substitute the free energy with the elastic energy $e(F, A, \nabla A)$. If we consider a linear elastic behavior in the range of infinitesimal deformations, by substituting $F$ with the gradient of the displacement $u$, namely $\nabla u$, the simplest expression of $e(\nabla \mathrm{u}, \mathrm{A}, \nabla \mathrm{A})$ is given by

$$
e(\nabla \mathrm{u}, \mathrm{A}, \nabla \mathrm{A})=0,5(\mathrm{C} \nabla \mathrm{u}) \cdot \nabla \mathrm{u}+\left(\mathrm{D}_{1} \mathrm{~A}\right) \cdot \nabla \mathrm{u}+0,5\left(\mathrm{D}_{2} \mathrm{~A}\right) \cdot \mathrm{A}+0,5\left(\mathrm{D}_{3} \nabla \mathrm{A}\right) \cdot \nabla \mathrm{A}
$$

where $C, D_{1}, D_{2}$ are fourth-order tensors and $D_{3}$ is a sixth-order tensor.

\section{CONSTITUTIVE PRESCRIPTIONS FROM LATTICES}

We identify the explicit expression of the constitutive tensors in (11) from a complex lattice by means of an identification procedure based on an equivalence in terms of power, following suggestions of [7]. We consider a complex two-level periodic lattice with characteristic cell shown in Figure 1. The cell is the "model" of the material element and through the identification procedure we attribute all the properties of the cell to every point in B. The complex lattice is made of two superposed lattices connected by elastic links: the first one made of material points (the black spheres in Fig. 1) the other one by four sub-lattices. In the whole cell the links can carry only axial forces and we write $t$ for the interactions in the first lattice (links between black spheres), $t^{\circ}$ for the interactions between the two lattices (diagonal links), $t^{\star}$ for the interactions between sub-lattices, $t^{\prime \prime}$ for interactions in each sub-lattice. We also indicate by $i, j, \ldots$ the placements of the black 
spheres, by $r, s, \ldots$ the end points of each link between sub-lattices and with $h, k, \ldots$ the placements of the spheres in the sub-lattices, and by $u^{i}$ the displacement of the point at $x^{i}$ in the lattice (the same notations hold for all other indices).

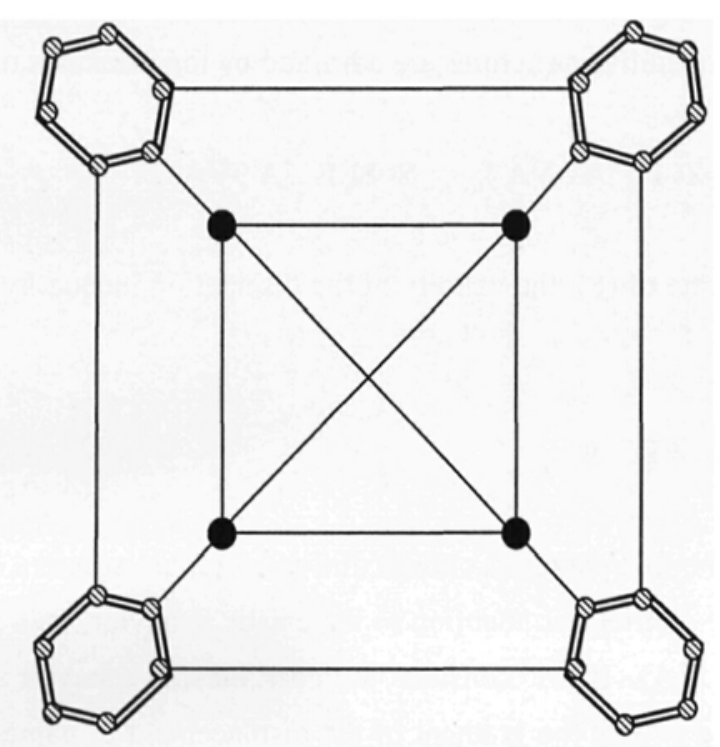

Fig. 1: Characteristic cell of the two-level lattice.

Appropriate measures of deformation of the lattice are then the following ones:

$$
\Delta=l^{-1}\left(\mathrm{u}^{\mathrm{i}}-\mathrm{u}^{\mathrm{j}}\right) ; \Delta^{\text {in }}=\left(l^{\prime \prime}\right)^{-1}\left(\mathrm{u}^{\mathrm{s}}-\mathrm{u}^{\mathrm{i}}\right) ; \Delta^{\text {ful }}=\left(l^{\prime \prime l}\right)^{-1}\left(\mathrm{u}^{\mathrm{h}}-\mathrm{u}^{\mathrm{k}}\right) ; \Delta^{\text {if }}=\left(l^{\prime}\right)^{-1}\left(\mathrm{u}^{\mathrm{s}}-\mathrm{u}^{\prime}\right)
$$

where $\Delta$ is the elongation of the generic rod of initial length $l$ between black spheres in the first lattice, $\Delta^{\text {in }}$ is referred to interlattice links, $\Delta^{\text {ful }}$ to the links in the sub-lattice and, finally, $\Delta^{\text {df }}$ to the links between sublattices. The power $\pi$ in the cell is thus

$$
\pi=\Sigma_{\mathrm{L}}(\mathrm{t} \cdot \Delta)+\Sigma_{\mathrm{Li}}\left(\mathrm{t}^{\circ} \cdot \Delta^{\mathrm{in}}\right)+\Sigma_{\mathrm{Lf}}\left(\mathrm{t}^{\cdot} \cdot \Delta^{\mathrm{ful}}\right)+\Sigma_{\mathrm{Lff}}\left(\mathrm{t}^{\#} \cdot \Delta^{\mathrm{uf}}\right)
$$

where $\Sigma_{\mathrm{L}}$ indicates the summation over the number of links between black spheres, while $\Sigma_{\mathrm{Li}}, \Sigma_{\mathrm{Lf}}, \Sigma_{\mathrm{Lif}}$ have analogous meaning with reference to the interlattices links, the ones in the sublattices and the ones between sub-lattices. The basic point of the procedure is the following identification

$$
\pi=V_{\mathrm{RVE}}(\mathrm{T} \cdot \mathrm{F}+\mathrm{Z} \cdot \mathrm{A}+\mathrm{S} \cdot \nabla \mathrm{A})
$$


where $V_{\mathrm{RVE}}$ is the volume of the smallest convex region containing the cell. To find explicitly the constitutive tensors we need (i) to express the $\Delta^{\prime}$ 's in terms of $\nabla u, A$ and $\nabla A$ and (ii) to assign some constitutive relations for the t's. For step (i) we assume that the first lattice (the one made of black spheres in Figure 1) undergoes only homogeneous deformations and that the sub-lattices undergo also homogeneous deformations but the deformation of one sub-lattice is different (in principle) from the deformation of the neighboring one. The links between $\Delta$ 's and of $\nabla \mathrm{u}, \mathrm{A}$ and $\nabla \mathrm{A}$ follow immediately. We also assume that the interactions in the lattice have the following constitutive structure:

$$
\mathbf{t}=\mathrm{K} \Delta ; \mathbf{t}^{\circ}=\mathrm{K}^{\circ} \Delta^{\text {in }} ; \mathbf{t}^{*}=\mathrm{K}^{*} \Delta^{\text {ful }} ; \mathbf{t}^{\prime \prime}=\mathrm{K}^{\#} \Delta^{\text {if }}
$$

As a consequence, we find

$$
\begin{aligned}
& V_{R V E} C=\Sigma_{L}\left(\left(x^{i}-x^{j}\right) \otimes K \otimes\left(x^{i}-x^{j}\right)\right)+\Sigma_{L i}\left(\left(x^{i}-x^{\prime}\right) \otimes K^{\circ} \otimes\left(x^{i}-x^{\prime}\right)\right), \\
& V_{R V E} D_{1}=\Sigma_{L i}\left(\left(x^{s}-x^{\prime}\right) \otimes K^{\circ} \otimes\left(x^{i}-x^{\prime}\right)+\left(x^{i}-x^{\prime}\right) \otimes K^{\circ} \otimes\left(x^{s}-x^{\prime}\right)\right), \\
& V_{R V E} D_{2}=\Sigma_{L r}\left(\left(x^{h}-x^{k}\right) \otimes K^{*} \otimes\left(x^{h}-x^{k}\right)\right)+\Sigma_{L i}\left(\left(x^{s}-x^{\prime}\right) \otimes K^{\circ} \otimes\left(x^{s}-x^{\prime}\right)\right), \\
& V_{R V E} D_{3}=\Sigma_{L, f}\left(\left(x^{s}-x^{\prime}\right) \otimes\left(x^{s}-x^{i}\right) \otimes K^{\prime \prime} \otimes\left(\left(x^{s}-x^{\mathrm{j}}\right) \otimes\left(x^{s}-x^{j}\right)\right),\right.
\end{aligned}
$$

where $x^{\prime}$ is an arbitrary point chosen appropriately in the cell. The constitutive tensors depend on the mechanical properties of the lattice (the $\mathrm{K}$ 's), at least as regards the linear approximation, and the geometry of it.

Formulas (16)-(19) permit the development of concrete algorithms for computations that could show the influence of the presence of the fullerenes on the macroscopic strain, generating possibly strain localization zones when the matrix of the composite is softer than the fullerenes.

\section{REFERENCES}

[1] Kim, H., Kim J. Y., Lee, K., Park, Y., Jin, Y. and Suh, H., Organic photovoltaic cells based on conjugated polymer/fullerene composites, Curr. Appl. Phys., 1, 139-143 (2001).

[2] Mordkovich, V. Z. and Takeuchi, Y., Multishell fullerenes by laser vaporization of composite carbonmetal targets, Chem. Phys. Letters, 355, 133-138 (2002).

[3] Kadish, K. M. and Ruoff, R. S. (Eds), Fullerenes, Wiley, New York (2000).

[4] Capriz, G., Continua with microstructure, Springer-Verlag, Berlin (1989). 
[5] Capriz, G. and Mariano, P. M. (Eds), Advances in multifield theories for continua with substructure, Birkhauser, Boston (2003).

[6] Mariano, P. M., Multifield theories in mechanics of solids, Adv. Appl. Mech., 38, 1-93 (2002).

[7] Atyiah, M. and Sutcliffe, P., The geometry of point particles, R. Soc. Lon. Proc. Ser. A Math. Phys. Eng. Sci., 458, 1089-1115 (2002).

[8] Mariano, P. M. and Stazi, F. L., Strain localization in elastic microcracked bodies, Comp. Meth. Appl. Mech. Eng., 190, 5657-5677 (2001). 